\title{
Solvation Dynamics of Coumarin 480 in Sol-Gel Matrix
}

\author{
Samir Kumar Pal, Dipankar Sukul, Debabrata Mandal, Sobhan Sen, and \\ Kankan Bhattacharyya*
}

Physical Chemistry Department, Indian Association for the Cultivation of Science, Jadavpur, Calcutta 700 032, India

Received: September 29, 1999; In Final Form: December 15, 1999

\begin{abstract}
Solvation dynamics of Coumarin 480 (C-480) in a tetraethyl orthosilicate (TEOS) sol-gel matrix has been studied using time-resolved emission spectroscopy. In the macroscopically solid TEOS matrix the solvation dynamics of C-480 is described by a major (85\%) component of $120 \pm 20 \mathrm{ps}$ and a minor (15\%) component of $800 \pm 100$ ps. These components are substantially slower than the solvation dynamics in bulk water. The rotational relaxation time of $\mathrm{C}-480$ in this matrix is found to be very short $(<80 \mathrm{ps})$.
\end{abstract}

\section{Introduction}

Water molecules in confined environments play a crucial role in many natural processes and control the structure, function, and dynamics of many biomolecules. Recently, several groups studied the dynamics of water and other small molecules in a wide variety of confined environments. ${ }^{1-11}$ These include water surface, ${ }^{1,4}$ proteins, ${ }^{2}$ cyclodextrin, ${ }^{3}$ microemulsion, ${ }^{5}$ sol-gel matrix, ${ }^{6,7}$ zirconia particle,${ }^{8}$ polymer hydrogel,,${ }^{9}$ lipid,,${ }^{10}$ and micelles. ${ }^{11}$ The dielectric relaxation time of pure water is 10 ps. ${ }^{12}$ In bulk water, solvation dynamics occurs on the subpicosecond time scale. ${ }^{2,13}$ However, in most of these organized and confined media both solvation dynamics ${ }^{3,5,10-11}$ and dielectric relaxation ${ }^{2,14}$ of water are found to be slower by 3-4 orders of magnitude. In the case of a microporous solid (e.g., sol-gel matrix $^{6,7}$ or polymer hydrogel ${ }^{9}$ ) the bulk viscosity is very high and, hence, a very slow relaxation dynamics is expected. However, recently it is reported that both the sol-gel ${ }^{6}$ and polymer hydrogel ${ }^{9}$ exhibit very fast solvation dynamics and rotational relaxation. The fast dynamics is attributed to the presence of large pores in these media. ${ }^{15,16}$ Large biomolecules can pass through these pores, and hence, the dynamics of small water molecules or fluorescent probes is very fast in these media. The inorganic sol-gel composite obtained from the hydrolysis of tetraalkyl orthosilicate acts as a good host for many biological materials. ${ }^{17}$ Many enzymes such as glucose oxidase, ${ }^{17 a}$ aspartase, ${ }^{17 \mathrm{~b}}$ urease, ${ }^{17 \mathrm{c}}$ and nonenzymes such as bacteriorhodopsin ${ }^{17 \mathrm{~d}}$ can be encapsulated in biologically active form for a very long period in a sol-gel glass. Such sol-gel glasses doped with biomolecules have potential applications as chemical sensors. It is obviously interesting to find out the dynamics occurring in such an interesting material. However, there have been relatively few studies on relaxation dynamics in sol-gel matrices. Bright et al. ${ }^{6}$ studied relaxation of acrylodan-labeled BSA in a sol-gel matrix using phase fluorometry and reported that the protein molecule remains highly mobile in this matrix. Pant and Levinger employed femtosecond upconversion to study the solvation dynamics of coumarin-343 (C-343) adsorbed to zirconia particles in water. ${ }^{8}$ Fourkas and co-workers ${ }^{7}$ carried out a careful study on the optical Kerr effect (OKE) of methyl iodide and acetonitrile in sol-gel glasses of different pore sizes.

*E-mail: pckb@mahendra.iacs.res.in. Fax: (91)-33-473-2805.

\section{SCHEME 1: Structure of Coumarin-480}

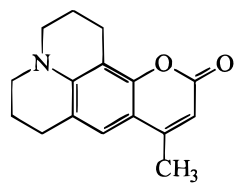

They observed that for both the liquids the decay of the OKE signal in a sol-gel glass is multiexponential with a major component similar to that in bulk liquid and an additional component, which is about 4 times slower. The ratio of the amplitudes of the fast (bulk) and the slow component increases with the pore size. ${ }^{7}$ In this work, we report on a picosecond time dependent Stokes shift (TDSS) study of solvation dynamics of water molecules trapped in a tetraethyl orthosilicate (TEOS) sol-gel matrix using coumarin 480 (C-480, Scheme 1) as a probe.

\section{Experimental Section}

TEOS (Aldrich, 99\%) and C-480 (Exciton) were used as received. To $1 \mathrm{~mL}$ of a $3 \times 10^{-4} \mathrm{M}$ solution of $\mathrm{C}-480$ in neat liquid TEOS taken in a quartz tube was added $1 \mathrm{~mL}$ of a $2.5 \times$ $10^{-3} \mathrm{~N}$ aqueous $\mathrm{HCl}$ solution drop by drop. The mixture is vortexed for $30 \mathrm{~min}$ and then allowed to stand at $40{ }^{\circ} \mathrm{C}$ for several hours to facilitate hydrolysis. Once the gel is formed, the gel was kept exposed to air for nearly 2 months to allow the alcohol formed to escape and to complete aging of the gel. The reported pore size of the sol-gel glass prepared at $\mathrm{pH}=$ 3 and $\mathrm{H}_{2} \mathrm{O} / \mathrm{Si}$ ratio $\approx 12$, is $10-20 \AA .{ }^{18}$ The absorption (against water as reference) and emission and excitation spectra of the gel were recorded periodically. It is observed that after 1 month the spectra display no further change. All steady state and timeresolved studies were made using a sufficiently aged gel (2 months old). The sol-gel matrix was found to cause scattering. At the wavelength of excitation $(300 \mathrm{~nm})$, due to scattering transmittance of the sol-gel sample without C-480 is $50 \%$. The sol-gel matrix, however, exhibited no emission.

Steady-state absorption and emission spectra were recorded on JASCO 7850 and Perkin-Elmer 44B instruments, respectively. Quantum yields $\left(\phi_{\mathrm{f}}\right)$ were measured using the reported ${ }^{19}$ quantum yield (0.66) of C-480 in water. The sample was excited at $300 \mathrm{~nm}$ with the second harmonic of a cavity-dumped 


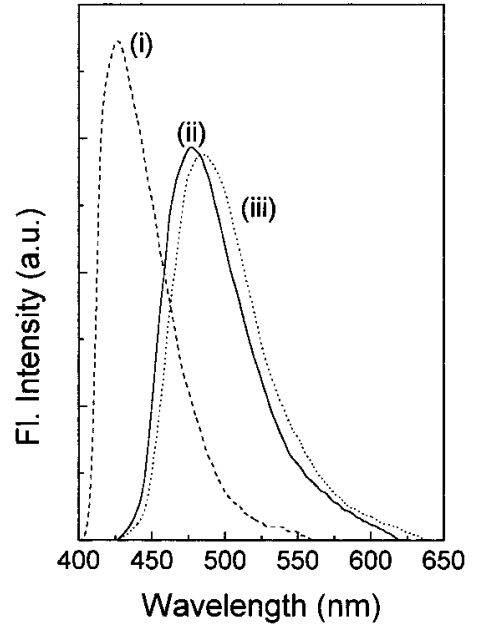

Figure 1. Emission spectra of C-480 in (i) neat TEOS (- - -), (ii) TEOS gel $(-)$, and (iii) water $(\cdots)$.

Rhodamine 6G dye laser (Coherent 702-1) pumped by a continuous wave (cw) mode-locked Nd:YAG laser (Coherent, Antares). The emission was collected at magic angle polarization for lifetime measurement by a Hamamatsu MCP photomultiplier (2809-U). The typical system response at $300 \mathrm{~nm}$ excitation is about 80 ps. For rotational relaxation studies, the emission intensity at perpendicular $\left(I_{\perp}\right)$ and parallel $\left(\mathrm{I}_{\|}\right)$polarizations were collected alternatively for $100 \mathrm{~s}$. For a typical anisotropy decay a peak count of 10000 counts was collected at parallel polarization. The $r(t)$ is then calculated using the relation

$$
r(t)=\frac{I_{\|}(t)-G I_{\perp}(t)}{I_{\|}(t)+2 G I_{\perp}(t)}
$$

The $G$ factor of the setup was determined using a dye whose rotational relaxation time is very short (e.g., nile red in methanol).

\section{Results}

3.1. Steady-State Spectra. In neat liquid TEOS, C-480 exhibits an absorption maximum at $365 \mathrm{~nm}$ and an intense emission with emission maximum at $430 \mathrm{~nm}$ (Figure 1) and quantum yield $\phi_{\mathrm{f}}=0.55$. The position of the maximum of C-480 in TEOS is intermediate between those reported ${ }^{19}$ in cyclohexane $\left(\lambda^{\mathrm{max}}{ }_{\mathrm{abs}}=360 \mathrm{~nm}\right.$ and $\left.\lambda^{\mathrm{max}}{ }_{\mathrm{em}}=410 \mathrm{~nm}\right)$ and acetonitrile $\left(\lambda^{\max }{ }_{a b s}=380 \mathrm{~nm}\right.$ and $\left.\lambda^{\max }{ }_{\text {em }}=450 \mathrm{~nm}\right)$. In the TEOS solgel matrix, the emission spectrum of C-480 exhibits a marked red shift to $480 \mathrm{~nm}$ (Figure 1) with $\phi_{\mathrm{f}}=0.55$ and the absorption spectrum (Figure 2) also exhibits a red shift to $390 \mathrm{~nm}$. The excitation spectrum of $\mathrm{C}-480$ in the gel (Figure 2) remains identical to the absorption spectrum, which rules out the presence of any impurity. It is evident that the absorption and emission maxima of C-480 in a gel are very similar to those in water ${ }^{19}\left(\lambda^{\max }{ }_{a b s}=395 \mathrm{~nm}\right.$ and $\left.\lambda^{\max }{ }_{\mathrm{em}}=490 \mathrm{~nm}\right)$. This indicates that in the TEOS gel C-480 experiences a highly polar and protic environment presumably due to the presence of a large amount of entrapped water.

3.2. Time-Resolved Study. In neat liquid TEOS, C-480 exhibits a single-exponential decay with lifetime $3.1 \mathrm{~ns}$ (Figure 3 ) and the decay is independent of the emission wavelength. The lifetime of C-480 in neat liquid TEOS is close to that reported ${ }^{19}$ in acetonitrile (3.3 ns). However, in the TEOS gel, the emission decays of C-480 are found to be wavelength dependent. At the red end the decay is preceded by a growth,

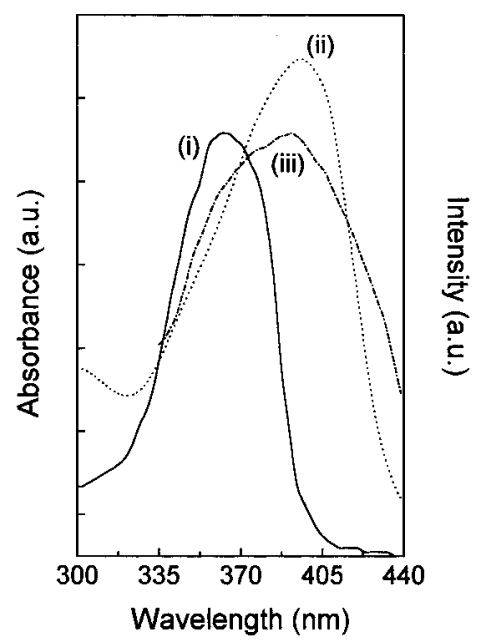

Figure 2. Absorption spectra of C-480 in (i) neat TEOS (-) and (ii) TEOS gel $(\cdots)$ and (iii) excitation spectrum of C-480 in TEOS gel $(-\cdot-)$.

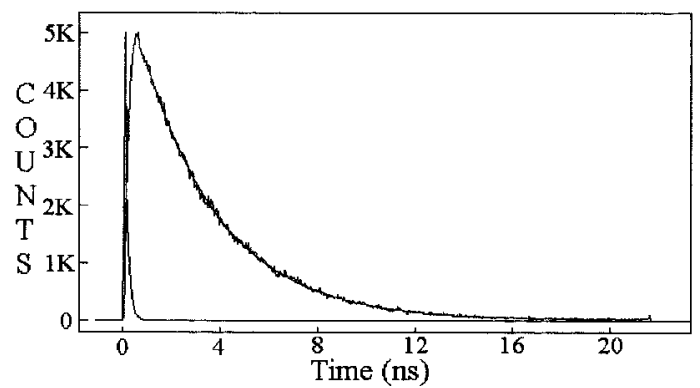

Figure 3. Fluorescence decay of C-480 in neat TEOS.

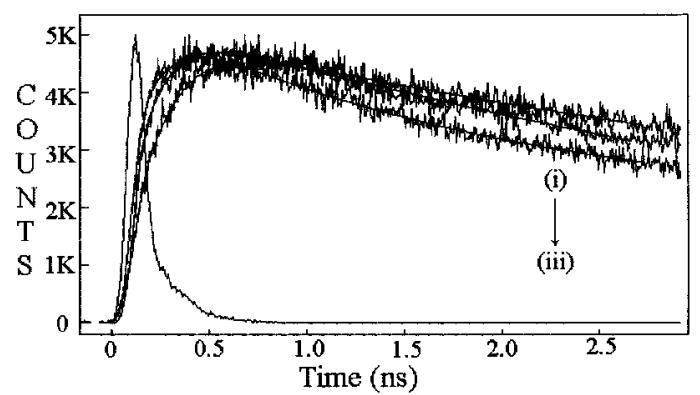

Figure 4. Fluorescence decays of C-480 in TEOS gel at (i) $550 \mathrm{~nm}$, (ii) $480 \mathrm{~nm}$, and (iii) $435 \mathrm{~nm}$.

while at the blue end a fast decay is observed (Figure 4). For example, the decay at the red end $(570 \mathrm{~nm})$ is fitted to a biexponential with a growth component of 200 ps and a decay component of $6.1 \mathrm{~ns}$ while at the blue end $(435 \mathrm{~nm})$ a biexponential decay with an average lifetime of $3.8 \mathrm{~ns}$ is observed. Such a decay at the blue end and growth at the red end is typical of systems undergoing solvation dynamics. ${ }^{20,21}$ From the decays the time-resolved emission spectra (Figure 5) were constructed by following the method discussed by Maroncelli and Fleming. ${ }^{20}$ The solvation dynamics is described by the decay of the solvent correlation function, $C(t)$, defined as

$$
C(t)=\frac{v(t)-v(\infty)}{v(0)-v(\infty)}
$$

where $v(0), v(t)$, and $v(\infty)$ are respectively the emission frequencies at time $0, t$, and $\infty$. The decay of $C(t)$ is shown in Figure 6, and the decay parameters are summarized in Table 1. It is readily seen that C-480 displays a fast solvation dynamics 


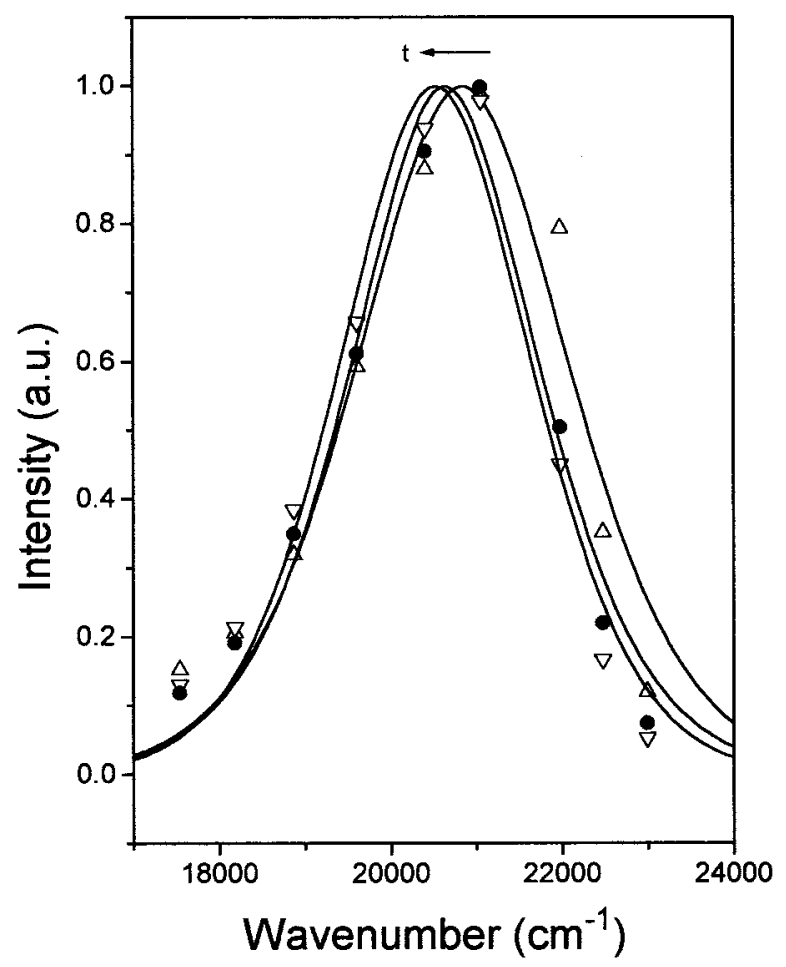

Figure 5. Time-resolved emission spectra of C-480 in TEOS gel at (i) 0 ps $(\triangle)$, (ii) $200 \mathrm{ps}(\bullet)$, (iii) $2000 \mathrm{ps}(\nabla)$.

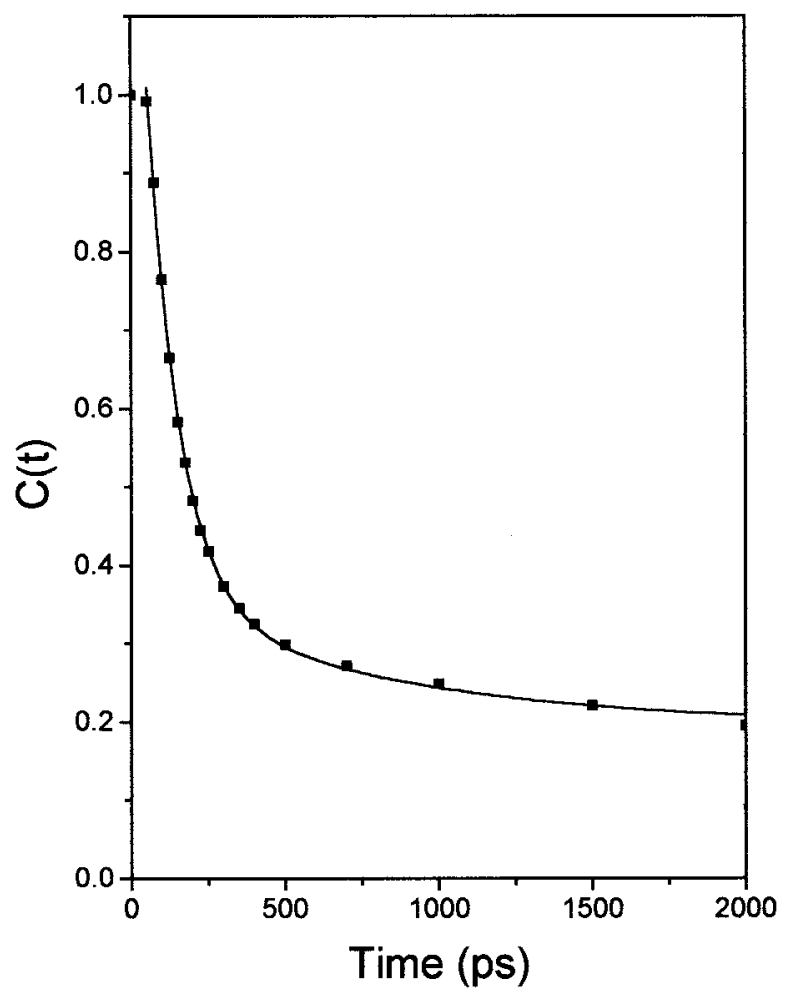

Figure 6. Decay of response function, $C(t)$, of C-480 in TEOS gel. The points denote the actual values of $C(t)$ and the solid line denotes the best fit to a biexponential decay.

with a major (85\%) component of $120 \pm 20$ ps and a minor (15\%) component of $800 \pm 100 \mathrm{ps}$, leading to an average solvation time $\left\langle\tau_{\mathrm{s}}\right\rangle=a_{1} \tau_{1}+a_{2} \tau_{2}=220 \pm 30 \mathrm{ps}$.

3.3. Rotational Relaxation. While solvation dynamics arises due to the motion of the trapped water molecules in the TEOS gel, the motion of the probe $\mathrm{C}-480$ gives rise to the time dependent optical anisotropy. It is observed that in the TEOS
TABLE 1: Decay Characteristics of the Solvent Response Function, $C(t)$, of $\mathrm{C}-480$ in TEOS Gel

\begin{tabular}{cccccc}
\hline$\Delta v\left(\mathrm{~cm}^{-1}\right)$ & $a_{1}$ & $\tau_{1}{ }^{a}(\mathrm{ps})$ & $a_{2}$ & $\tau_{2}{ }^{b}(\mathrm{ps})$ & $\left\langle\tau_{\mathrm{s}}{ }^{c}(\mathrm{ps})\right.$ \\
\hline 400 & 0.85 & 120 & 0.15 & 800 & 220 \\
${ }^{a} \pm 20$ ps. $^{b} \pm 100$ ps. ${ }^{c}\left\langle\tau_{\mathrm{s}}\right\rangle=a_{1} \tau_{1}+a_{2} \tau_{2} ; \pm 30$ ps. &
\end{tabular}

gel the rotational relaxation of $\mathrm{C}-480$, i.e., the decay of $r(t)$, is very fast and occurs in a time scale $<80$ ps.

\section{Discussion}

It is evident that the emission properties of C-480 in TEOS gel are substantially different from those in neat liquid TEOS. In the macroscopically solid gel, the probe C-480 molecule experiences a very polar environment, as indicated by the absorption and emission maxima, the quantum yield of emission, and the long component of decay of about $6 \mathrm{~ns}$, which is similar to the lifetime of $\mathrm{C}-480$ in water $\left(5.9 \mathrm{~ns}^{19}\right)$. The polar environment within the TEOS matrix may be attributed to the presence of trapped water. It is apparent that even in the rigid sol-gel matrix, the solvation dynamics is very fast. The average solvation time in the gel is $220 \pm 30$ ps. This is slower than the solvation time in bulk water $(0.3 \mathrm{ps}) .{ }^{3,13}$ However, the dynamics in the gel is much faster than the nanosecond dynamics observed in cyclodextrin, ${ }^{3}$ microemulsions, ${ }^{5} \mathrm{mi}$ celles, ${ }^{11}$ or lipids. ${ }^{10}$ The rotational relaxation study also suggests that the probe $\mathrm{C}-480$ remains highly mobile within the sol-gel matrix. It may be recalled that steady state anisotropy in a titania gel indicates that though the bulk viscosity is extremely high, the local microviscosity is extremely low. ${ }^{22}$ A previous phase fluorometry study in the sol-gel matrix ${ }^{6}$ and a TDSS study in the polyacrylamide hydrogel ${ }^{9}$ also suggest high mobility of organic fluorescent probes inside the gel. According to NMR ${ }^{23 a}$ and simulation ${ }^{23 b}$ studies the diffusion coefficient of water in a hydrogel is lower than that in bulk water only by a factor of 2 . Similar high mobility in the polyacrylamide hydrogel is also reported by Moerner et al. ${ }^{16}$ Using fluorescence microscopy, they demonstrated that almost all $(98 \%)$ of the probe molecules (nile red) remain highly mobile in the polyacrylamide hydrogel. ${ }^{16}$

As discussed by Fee and Maroncelli, a large part of the ultrafast component of solvation is missed in a picosecond setup. ${ }^{21}$ If the sample is excited near its absorption maximum (about $400 \mathrm{~nm}$ in this case), the amount of solvation missed may be calculated using the procedure described by Fee and Maroncelli. ${ }^{21}$ Unfortunately, using the second harmonic of our Rhodamine 6G/DODCI dye laser, we excited the sample at 300 $\mathrm{nm}$, which is at the blue end of its absorption spectrum. For the wavelength of excitation in the present study $(300 \mathrm{~nm})$ the several stages of curve fitting suggested by Fee and Maroncelli ${ }^{21}$ is unlikely to give a good estimate of the missed part of the Stokes shift. However, according to Fleming et al. for C-480 about $50 \%$ of the estimated Stokes shift is missed even in a femtosecond upconversion setup. ${ }^{3 b}$ Using a femtosecond setup they determined $\Delta v$ for $\mathrm{C}-480$ to be $1340 \mathrm{~cm}^{-1}$ in water. ${ }^{3 \mathrm{~b}} \mathrm{In}$ the sol-gel matrix $\Delta v$ for C-480 is found to be $400 \mathrm{~cm}^{-1}$, which is one-third of that in water. For C-343, the femtosecond upconversion study carried out by Pant and Levinger ${ }^{8}$ indicates $\Delta v$ to be $150 \mathrm{~cm}^{-1}$ in the zirconia particle, which is nearly onefifth of that $\left(800 \mathrm{~cm}^{-1}\right)$ in water.

The most significant observation of this work is the observation of a very long component of solvation in the sol-gel matrix. The solvation time of C-480 in the sol-gel matrix $(220 \pm 30$ ps) is nearly 700 times slower compared to that in bulk water $\left(0.3 \mathrm{ps}^{3 \mathrm{~b}}\right)$ and is about 100 times slower than that $(0.24 \mathrm{ps})$ in 
zirconia particles. ${ }^{8}$ It should, however, be emphasized that we are missing a large part of the Stokes shift, which continues to occur on a subpicosecond time scale, and are observing only a long-lived component of the overall solvation dynamics. Fourkas et al. ${ }^{7}$ reported a very fast and almost bulklike OKE relaxation in the sol-gel glass. For instance, at $290.6 \mathrm{~K}$ for $\mathrm{CH}_{3} \mathrm{I}$ in a sol-gel glass of pore size $24 \AA$, they found that the contribution of the bulklike component is $87 \%$ while that of a 4 times slower component is only $13 \% .^{7 \mathrm{~b}}$ The slow component of solvent relaxation and the fast rotational dynamics, reported in the present study and the fast OKE relaxation in the sol-gel glass ${ }^{7}$ may be reconciled as follows. The pore size of the glasses used by Fourkas et al. ${ }^{7}$ are much bigger than the size of the small $\mathrm{CH}_{3} \mathrm{I}$ or $\mathrm{CH}_{3} \mathrm{CN}$ molecules, and hence, dynamics of the latter is largely unhindered in the porous glass. However, in our case, the pores $(10-20 \AA)^{18}$ are only slightly bigger than the probe C-480 molecule. Since the probe C-480 fills up the pore, the movement of the water molecules becomes highly hindered. The other possible cause of retardation of motion of water molecules is hydrogen bonding between the water molecules and the silicate network. It appears that like solvation dynamics, a major part of the rotational dynamics remains bulklike and very fast in the gel. This fast component presumably obscures observation of any slow component of rotational dynamics. It seems that the relatively free rotational movement and presence of a large amount of water within the TEOS gel allows the biomolecules to attain their native or biologically active structure. ${ }^{17}$

\section{Conclusion}

The present work shows that C-480 dye molecules experience a highly polar and protic environment within the solid TEOS sol-gel matrix, as evidenced by the position of the absorption and emission maxima, quantum yield of emission, and long emission lifetime. The TDSS study indicates that the average solvation time of C-480 in the sol-gel glass of pore size 10$20 \AA,{ }^{18}$ is $220 \pm 30$ ps. This component is about 700 times slower than that in bulk water ${ }^{3}$ and about 100 times slower than that in a zirconia particle ${ }^{8}$ but is about 1 order of magnitude faster than the slow solvation dynamics reported in cyclodextrin, ${ }^{3}$ microemulsions, ${ }^{5}$ lipids, ${ }^{10}$ and micelles. ${ }^{11}$ The rotational relaxation of C-480 in TEOS gel is observed to be very fast $(<80 \mathrm{ps})$. A major part of solvation and rotational dynamics of C-480 in the gel, however, continue to be very fast and missed by a picosecond setup. The high rotational mobility of the probe and the presence of trapped water molecules may be responsible for the biological activity of entrapped biomolecules in the solgel matrix.

Acknowledgment. Thanks are due to Council of Scientific and Industrial Research (CSIR), Government of India, for generous research grants. S.K.P., D.S., D.M., and S.S. thank CSIR for awarding fellowships.

\section{References and Notes}

(1) (a) Eisenthal, K. B. Chem. Rev. 1996, 96, 1343. (b) Zhu, S.-B.; Singh, S.; Robinson, G. W. Adv. Chem. Phys. 1994, 85, 627.

(2) (a) Nandi, N.; Bagchi, B. J. Phys. Chem. A 1998, 102, 8217. (b) Nandi, N.; Bagchi, B. J. Phys. Chem. B 1997, 101, 10954.

(3) (a) Nandi, N.; Bagchi, B. J. Phys. Chem. 1996, 100, 13914. (b) Vajda, S.; Jimenez, R.; Rosenthal, S. J.; Fidler, V.; Fleming, G. R.; Castner, E. W., Jr. J. Chem. Soc., Faraday Trans. 1995, 91, 867.

(4) Zimdars, D.; Dapad, J. I.; Eisenthal, K. B.; Heinz, T. F. Chem. Phys. Lett. 1999, 301, 112.

(5) (a) Lundgren, I. S.; Heitz, M. P.; Bright, F. V. Anal. Chem. 1995 , 67, 3775. (b) Sarkar, N.; Das, K.; Datta, A.; Das, S.; Bhattacharyya, K. J. Phys. Chem. 1996, 100, 10523. (c) Riter, R. E.; Undiks, E. P.; Kimmel, J. R.; Pant, D. D.; Levinger, N. E. J. Phys. Chem. B 1998, 102, 7931. (d) Shirota, H.; Horie, K. J. Phys. Chem. B 1999, 103, 1437. 2436.

(6) Jordan, J. D.; Dunbar, R. A.; Bright, F. V. Anal. Chem. 1995, 67,

(7) (a) Loughanne, B. J.; Farrer, R. A.; Scodinu, A.; Fourkas, J. T. J. Chem. Phys. 1999, 111, 5116. (b) Loughanne, B. J.; Fourkas, J. T. J. Phys. Chem. B 1998, 102, 10228

(8) Pant, D.; Levinger, N. E. J. Phys. Chem. B 1999, 103, 7846.

(9) Datta, A.; Das, S.; Mandal, D.; Pal, S. K.; Bhattacharyya, K. Langmuir 1997, 13, 6922.

(10) Datta, A.; Pal, S. K.; Mandal, D.; Bhattacharyya, K. J. Phys. Chem. B 1998, 102, 6114 .

(11) Sarkar, N.; Datta, A.; Das, S.; Bhattacharyya, K. J. Phys. Chem. 1996, 100,15483

(12) Kaatze, U. Chem. Phys. Lett. 1993, 203, 1.

(13) Jimenez, R.; Fleming, G. R.; Kumar, P. V.; Maroncelli, M. Nature 1994, 370, 263.

(14) (a) Fukuzaki, M.; Miura, N.; Sinyashiki, N.; Kunita, D.; Shioya, S.; Haida, M.; Mashimo, S. J. Phys. Chem. 1995, 99, 431. (b) Belton, P. S. J. Phys. Chem. 1995, 99, 17061. (c) Denisov, V. P.; Peters, J.; Horlein, H. D.; Halle, B. Nature Struct. Biol. 1996, 3, 505.

(15) (a) Chrambach, A.; Rodbard, D. Science 1971, 172, 440. (b) Bromberg, L.; Grossberg, A. Yu.; Suzuki, Y.; Tanaka, T. J. Chem. Phys. 1997, 106, 2906.

(16) Dickson, R. M.; Norris, D. J.; Tzeng, Y.-L.; Moerner, W. E. Science 1996, $274,966$.

(17) (a) Narang, U.; Prasad, P. N.; Bright, F. V.; Kumar, A.; Kumar, N. D.; Malhotra, B. D.; Kamalasanan, M. N.; Chandra, S. Anal. Chem. 1994, 66, 3139. (b) Avnir, D.; Braun, S.; Ottolenghi, M. In Supramolecular Architecture; Bein, T., Ed.; ACS Symposium Series 499; American Chemical Society: Washington, DC, 1992. (c) Narang, U.; Prasad, P. N.; Bright, F. V.; Kumar, A.; Kumar, N. D.; Malhotra, B. D.; Kamalasanan, M. N.; Chandra, S. Chem. Mater. 1994, 6, 1596. (d) Wu, S.; Ellerby, M.; Cohan, J. S.; Dunn, B.; El-Sayed, M. A.; Valentine, J. S.; Zink, J. I. Chem. Mater. 1993, 5, 115. (e) Avnir, D.; Levy, D.; Reisfield, R. J. Phys. Chem. 1984, 88, 5956. (f) Unger, K. Porous Silica; Elsevier: Amsterdam, 1979. (g) Binker, C. J.; Scherer, G. W. Sol-Gel Science; Academic Press: CA, 1990.

(18) (a) Binker, C. J.; Keefer, K. D.; Schaffer, D. W.; Ashley, C. S. J. Non-Cryst. Solids 1982, 48, 47. (b) Krol, D. M.; van Lierop, J. G. J. NonCryst. Solids 1984, 63, 131.

(19) Jones, G., II; Jackson, W. R.; Choi, C.-Y.; Bergmark, W. R. J. Phys. Chem. 1985, 89, 294.

(20) Maroncelli, M.; Fleming, G. R. J. Chem. Phys. 1987, 86, 6221.

(21) Fee, R. S.; Maroncelli, M. Chem. Phys. 1994, 183, 235.

(22) Claudia-Marchi, M.; Bilmes, S. A.; Negri, R. Langmuir 1997, 13 , 3655 .

(23) (a) Hsu, T.-P.; Ma, D. S.; Cohen, C. Polymer 1983, 24, 1273. (b) Netz, P. A.; Dorfmuller, T. J. Phys. Chem. B 1998, 102, 4875. 